\title{
El registro más septentrional de la libélula gigante neotropical Megaloprepus caerulatus (Drury, 1782) (Odonata: Coenagrionidae) en el continente Americano
}

\section{The northernmost record of the Neotropical giant damselfly Megaloprepus caerulatus (Drury, 1782) (Odonata: Coenagrionidae) in the American continent}

\author{
Jaime A. Escoto-Moreno ${ }^{1}$, Alba Hernández-Hernández ${ }^{2}$, Jesús A. Hernández-Hernández ${ }^{2}$, Juan \\ MÁrquez ${ }^{3, *}$, Marcelo Silva-Briano ${ }^{4} \&$ Rodolfo Novelo-Gutiérrez ${ }^{5}$
}

${ }^{1}$ Colección Zoológica, Departamento de Biología, Centro de Ciencias Básicas, Universidad Autónoma de Aguascalientes. Avenida Universidad \# 940. Ciudad Universitaria, 20131 Aguascalientes, Aguascalientes, México. jerjaem2002@yahoo.es ${ }^{2}$ Laboratorio de Biología, Instituto Tecnológico de Huejutla, Tecnológico Nacional de México, km 5.5 carretera HuejutlaChalahuiyapa s/n, 43000 Huejutla de Reyes, Hidalgo, México. E-mail: (AH) albita_22@outlook.es; (JA) j22alberto@outlook. com

${ }^{3}$ Laboratorio de Sistemática Animal, Centro de Investigaciones Biológicas, Universidad Autónoma del Estado de Hidalgo, km 4.5 carretera Pachuca-Tulancingo s/n, Ciudad del Conocimiento, Col. Carboneras, 42184 Mineral de la Reforma, Hidalgo, México.

${ }^{4}$ Laboratorio de Ecología No. 1, Departamento de Biología, Centro de Ciencias Básicas, Universidad Autónoma de Aguascalientes. Avenida Universidad \# 940. Ciudad Universitaria, 20131 Aguascalientes, Aguascalientes, México. msilva@ correo.uaa.mx

${ }^{5}$ Red de Biodiversidad y Sistemática, Instituto de Ecología, A. C. Carretera antigua a Coatepec \# 351, El Haya, 91070 Xalapa, Veracruz, México. rodolfo.novelo@inecol.mx

*E-mail: marquezorum@gmail.com

\section{RESUMEN}

Megaloprepus caerulatus (Drury, 1782) es una de las libélulas más grandes en el mundo y se registra aquí por primera vez en Acomul, Hidalgo, México, siendo éste el registro más septentrional conocido en el Continente Americano, lo que permite discutir su distribución geográfica actual y su estatus taxonómico.

\begin{abstract}
Megaloprepus caerulatus (Drury, 1782) is one of the largest damselflies in the world and it is recorded here for the first time in Acomul, state of Hidalgo, Mexico, being this the northernmost record known in the American Continent, which allows us to discuss its current geographic distribution and its taxonomic status.
\end{abstract}

Megaloprepus caerulatus (Drury, 1782) es actualmente la única especie conocida del género. En la mayoría de las medidas corporales es la libélula más grande existente en el mundo (Feindt et al. 2014), con una envergadura alar de más de $190 \mathrm{~mm}$ y una longitud total de 89-120 mm (Esquivel 2006; Garrison et al. 2010). La especie ha sido estudiada desde el punto de vista ecológico y conductual en varios hábitats neotropicales (Fincke 1992, 1997).

La distribución conocida de $M$. caerulatus incluye una vasta zona de la región Neotropical que abarca desde Bolivia, Perú, Ecuador, Colombia, Guyana y Venezuela en América del Sur (Paulson 1983), todos los países de América Central
(Garrison et al. 2010), hasta el sur de México (Paulson \& González 1994) en los estados de Chiapas (Montebello), Oaxaca, Veracruz (Reserva de la Biósfera Los Tuxtlas) y Puebla (Zihuateutla) (Cuevas-Yañez 2007). Nunca ha sido encontrada en latitudes altas en la vertiente del Pacífico Mexicano.

Los géneros del Nuevo Mundo Mecistogaster, Microstigma, Pseudostigma, Anomisma y Megaloprepus antes estaban incluidos en la familia Pseudostigmatidae (Garrison et al. 2010), pero con estudios moleculares recientes de redefinición de las familias de libélulas, se observó que Coenagrionidae era una familia monofilética 
si Pseudostigmatidae y Protoneuridae del Nuevo Mundo eran incluidos (Dijkstra et al. 2014). Otro análisis del genoma mitocondrial completo de $M$. caerulatus sugiere que el hecho de contener cuatro regiones espaciadoras intergénicas, indica una posible característica específica de la familia Coenagrionidae y sus parientes cercanos (Feindt et al. 2016).

Feindt et al. (2014), usaron un análisis de estructura de poblaciones con dos marcadores de secuencias (ND1 y 16S rRNA) y un conjunto de microsatélites en poblaciones de la Isla Barro Colorado (Panamá), Parque Nacional Corcovado (Costa Rica), Estación de Investigación Biológica La Selva (Costa Rica) y la Reserva de la Biósfera de Los Tuxtlas en Veracruz, México (el registro más al norte conocido hasta ese momento), y los resultados muestran una ausencia de flujo genético y haplotipos no compartidos entre las poblaciones estudiadas. Asimismo, un estudio cladístico indica que el género Megaloprepus debería estar integrado por más de una especie (Feindt et al. 2014).

La finalidad de este trabajo es registrar por primera vez a $M$. caerulatus para el estado de Hidalgo, México, siendo éste el registro más septentrional en el continente Americano. Además, se discute la importancia de conocer lo mejor posible la distribución geográfica de la especie, con la finalidad de poder realizar un análisis genotípico integrativo en el continente Americano que ayude a clarificar el estatus taxonómico del género y brinde elementos para la conservación de sus poblaciones.

Se realizaron colectas de odonatos de mayo a diciembre de 2015 en el municipio de Xochiatipan, en el estado de Hidalgo; los sitios de colecta se ubican entre las coordenadas geográficas $20^{\circ} 46^{\prime}-20^{\circ} 57^{\prime} \mathrm{N}$ y $101^{\circ} 53^{\prime}-102^{\circ} 22^{\prime} \mathrm{W}$, los cuales tienen una humedad relativa alta, precipitación anual de 1,900 y 2,100 mm, un gradiente altitudinal de 200 a 1,000 $\mathrm{m}$, con temperaturas anuales promedio entre 20 y $24^{\circ} \mathrm{C}$. La vegetación presente es bosque tropical perennifolio (que casi ha desaparecido) y áreas de agricultura (VillavicencioNieto \& Pérez-Escandón 2010) y pertenece a la ecorregión bosques húmedos de Veracruz (Sánchez-Rojas \& BravoCadena 2017). Las capturas fueron realizadas usando una red aérea y los organismos colectados fueron sacrificados sumergiéndolos e inyectándoles acetona al 100\%. Los especímenes fueron depositados en la Colección Entomológica del Centro de Investigaciones Biológicas, Universidad Autónoma del Estado de Hidalgo (CE-UAEH), Hidalgo, México.

Material estudiado: 19 , México: Hidalgo, Xochiatipan, Acomul; N 20.809753 y W 98.315044; bosque tropical con dosel cerrado, 630 m, 24-IX-2015, J. A. HernándezHernández y A. Hernández-Hernández cols.

El ejemplar colectado posee una longitud total de 91 mm (Fig. 1). Este es el registro geográfico más septentrional conocido hasta el momento para esta especie, ya que los registros que se conocían eran la Reserva de la Biosfera de Los Tuxtlas, Veracruz que se ubica N 18.6000 y W 95.1500 y la localidad El Pozo, Zihuateutla, Puebla N 20.23944 y W 97.891667.

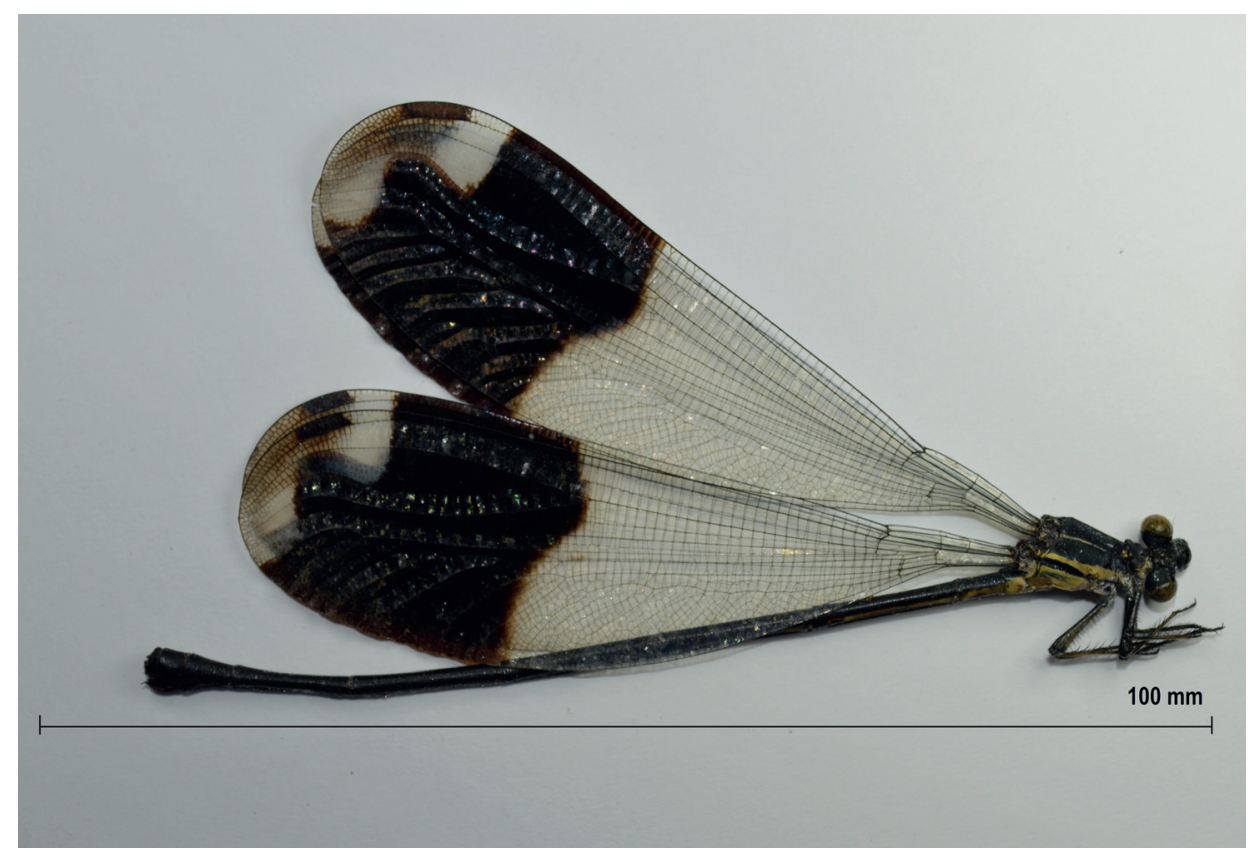

FIgURA 1. Vista lateral de la hembra de Megaloprepus caerulatus (foto A. Hernández-Hernández). / Lateral view of the female of Megaloprepus caerulatus (photo A. Hernández-Hernández). 
El hecho de haber encontrado un único ejemplar durante la época de lluvias (septiembre), a pesar de haber realizado un esfuerzo de recolecta total de 12 horas en siete localidades muestreadas durante ocho meses del municipio de Xochiatipan, sugiere hasta hoy una probabilidad baja de encontrar la especie y que su población posiblemente es reducida, dado que como adulto puede vivir más de ocho meses y es muy visible en los claros de los bosques, posando o volando cerca de los huecos de árboles con agua (Esquivel 2006), lo cual no sucedió. Sin embargo, no se descarta la posibilidad de buscar las larvas que pueden pasar de cuatro a ocho meses viviendo en los huecos de árboles con agua (Fincke 1996), lo cual daría más información y en el caso de que fueran abundantes proporcionaría suficiente material para los análisis moleculares.

Es importante mencionar que González et al. (2011) consideran a la Huasteca Potosina (sur del estado de San Luis Potosí) como el segundo hotspot más diverso de odonatos de México (el que se considera más diverso es el de Los Tuxtlas, Veracruz), basando esta conclusión en el análisis de especies de libélulas que ellos mismos colectaron, en adición a los registros históricos de 1957 a 1962 de la serie de expediciones que realizó George H. Beatty y Alice F. Beatty a la Huasteca Potosina. Dichos autores no encontraron nunca a Megaloprepus caerulatus en ese hotspot (hace 60 años el área estaba más conservada que hoy), ubicado a $70 \mathrm{~km}$ al norte de Acomul.

Por otra parte, la falta de estudios al norte de Veracruz y el sur de Tamaulipas, sitios relativamente cercanos a Acomul, así como los registros de otras libélulas gigantes neotropicales como Mecistogaster modesta, M. ornata y Pseudostigma aberrans en Tamaulipas (Paulson \& González 1994), sugieren una hipótesis: el límite septentrional de $M$. caerulatus está confinado a fragmentos de bosques tropicales en ecotonos entre las provincias biogeográficas de la Sierra Madre Oriental y Golfo de México (sensu Morrone 2005), ubicados al noreste del estado de Hidalgo, con la posibilidad de encontrar a la libélula en bosque similares al de Acomul en futuras expediciones al norte de Veracruz y sur de Tamaulipas, sin descartar algunos bosques de la parte sur-oriental del estado de San Luis Potosí, a pesar de que nunca se encontró en la Huasteca Potosina (González et al. 2011). Si se encontrase en estas áreas coincidiría con un nodo panbiogeográfico (lugar donde se mezclan elementos biogeográficos de diferente origen, por lo que se espera una alta diversidad) ya detectado con plantas y escarabajos (Luna et al. 2000, Márquez \& Morrone 2004).

Es importante conocer la distribución geográfica completa de $M$. caerulatus para generar un modelo de distribución de la especie que sirva como guía para la búsqueda de nuevas poblaciones en las áreas mencionadas. Después, se puede realizar un análisis del patrón genotípico de todas las poblaciones, incluyendo las más septentrionales de México (estados de Hidalgo y Puebla, así como las nuevas que pudieran aparecer en las expediciones en el norte de Veracruz, sur de Tamaulipas y oriente de San Luis Potosí), el cual permita decidir si se trata de una o más especies, con lo que se estaría sometiendo a prueba la hipótesis de Feindt et al. (2014).

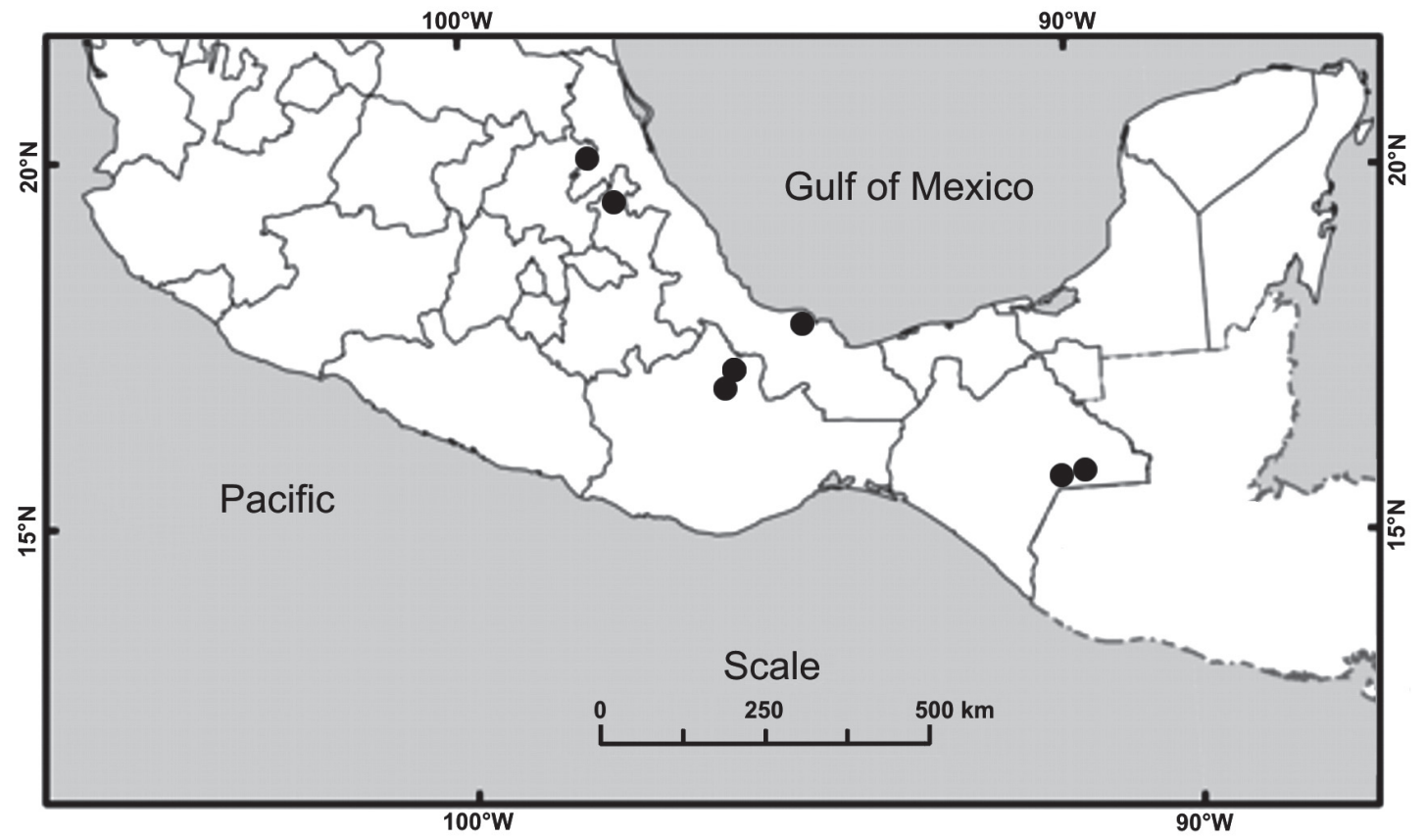

FiguRa 2. Registros geográficos de Megaloprepus caerulatus en México. / Geographic records of Megaloprepus caerulatus in Mexico. 
Una vez conocido lo anterior, se podrá tener certeza del estatus taxonómico del género, que es muy importante aclarar, porque las especies con mayor utilidad como indicadores de cambios en las condiciones ambientales deben tener una taxonomía bien conocida. Además, en el caso de bosques tropicales, las especies deben tener un rango de distribución moderado, habitando nichos extremadamente especializados, como Megaloprepus (Feindt et al. 2014).

Finalmente, es importante mencionar la acelerada deforestación y amenazas graves de destrucción del hábitat de esta especie por actividades humanas en México, por lo que esta libélula gigante y altamente susceptible debería tener prioridad para su conservación y ser incluida en la Norma Oficial Mexicana NOM-059-SEMARNAT-2010 (PROFEPA 2016) y en la IUCN Red List, especialmente por su continuo declive demográfico, ya comprobado en La Reserva de la Biosfera Los Tuxtlas en Veracruz, con una baja diversidad genética en los parches forestales, y además podría ser un útil indicador de cambios en la condiciones ambientales en los diferentes sitios de Mesoamérica como proponen Feindt et al. (2014).

\section{AGRADECIMIENTOS}

El segundo y tercer autor agradecen al Biólogo Raúl Valencia Herverth (Instituto Tecnológico de Huejutla) por su apoyo en las salidas de campo. Los autores agradecen a la M. en C. Julieta Asiain (CIB-UAEH) por su apoyo en la edición del mapa.

\section{REFERENCIAS}

Cuevas, K. 2007. Los odonatos (Insecta: Odonata) de la hidroeléctrica de Patla (El Pozo) y del río Tecpatlán, Zihuateutla, Puebla, México. Dugesiana 14(2):83-91.

Dijkstra, K.D.B., Kalkman, V.J., Dow, R.A., Stokvis, F.R., VAN Tol, J. 2014. Redefining the damselfly families: a comprehensive molecular phylogeny of Zygoptera (Odonata). Systematic Entomology 39:68-96.

Esquivel, C. 2006. Libélulas de Mesoamérica y el Caribe. Instituto Nacional de Biodiversidad (INBio), Costa Rica. 320 pp.

Feindt, W., Fincke, O. Hadrys, H. 2014. Still a one species genus? Strong genetic diversification in the world's largest living odonate, the Neotropical damselfly Megaloprepus caerulatus. Conservation Genetics 15:469-481.

Feindt, W., Osigus, H.J., Herzog, R., Mason, C.E., Hadrys, H. 2016. The complete mitochondrial genome of the neotropical helicopter damselfly Megaloprepus caerulatus (Odonata: Zygoptera) assembled from next generation sequencing data. Mitochondrial DNA Part B 1(1):497-499.

FincKe, O.M. 1992. Interspecific competition for tree holes: consequences for mating systems and coexistence in neotropical damselflies. The American Naturalist 139(1):80-101.

FINCKE, O.M. 1996. Larval behavior of a giant damselfly: territoriality or size-dependent dominance? Animal Behavior 51:77-87.

FINCKE, O.M. 1997. Conflict resolution in the Odonata: implications for understanding female mating patterns and female choice. Biological Journal of the Linnean Society 60:201220.

Garrison, R.W., von Ellenrieder, N., Louton, J.A. 2010. Damselfly genera of the New World. An illustrated and annotated key to the Zygoptera. The Johns Hopkins University Press Baltimore, Maryland, USA. 490 pp.

GonzÁlez, E., Noguera, F., OÑate, L. 2011. A biodiversity hotspot for Odonates in Mexico: The Huasteca Potosina, San Luis Potosí. Odonatologica 40(3):179-190.

Luna, I., Alcántara, O., Morrone, J.J., Espinosa, D. 2000. Track analysis and conservation priorities in the cloud forests of Hidalgo, Mexico. Diversity and Distributions 6:137-143.

Márquez, J., Morrone, J.J. 2004. Relaciones biogeográficas basadas en la distribución de Coleoptera (Insecta). En: Luna, I., Morrone, J.J., Espinosa, D. (Eds.) Biodiversidad de la Sierra Madre Oriental: 375-392. Las Prensas de la Ciencias, UNAM, México, D.F.

Morrone, J.J. 2005. Hacia una síntesis biogeográfica de México. Revista Mexicana de Biodiversidad 76(2):207-252.

Paulson, D.R. 1983. List of the Odonata of South America, by country. Slater Museum of Natural History, University of Puget Sound, Tacoma, Washington. URL: http:// www.pugetsound.edu/academics/academic-resources/ slater-museum/biodiversity-resources/dragonflies/southamerican-odonata/. Accedido: Noviembre 13, 2017.

Paulson, D.R., GonzÁlez, E. 1994. Odonata of Mexico by state. Slater Museum of Natural History, University of Puget Sound, Tacoma, Washington. URL: http://www. pugetsound.edu/academics/academic-resources/slatermuseum/biodiversity-resources/dragonflies/mexicanodonata/ Accedido: Noviembre 13, 2017.

PROFEPA. 2016. Norma Oficial Mexicana NOM-059SEMARNAT-2010. Categorías de riesgo y especificaciones para su inclusión, exclusión o cambio de lista de especies en riesgo. Procuraduría Federal de Protección al Ambiente. Diario Oficial, México. 78 pp.

Sánchez-Rojas, G., Bravo-Cadena, J. 2017. Medio físico del estado de Hidalgo. En: Ramírez-Bautista, A., SánchezGonzález, A., Sánchez-Rojas, G., Cuevas-Cardona, C. (Eds.) Biodiversidad del estado de Hidalgo (Tomo I): 29-42. Universidad Autónoma del Estado de Hidalgo Consejo Nacional de Ciencia y Tecnología. Pachuca de Soto, Hidalgo, México.

Villavicencio-Nieto, M.A., Pérez-Escandón, B.E. 2010. Vegetación e inventario de la flora útil de la Huasteca y la zona Otomí-Tepehua de Hidalgo. Ciencia Universitaria, UAEH 1:23-33. 